\title{
Interpolating Genetic Variation in Natural Populations: A Case Study of Plains Rough Fescue (Festuca hallii)
}

\author{
Yong-Bi Fu*,1, Jie Qiu ${ }^{1,2}$ and John F. Wilmshurst ${ }^{3}$ \\ ${ }^{I}$ Plant Gene Resources of Canada, Saskatoon Research Centre, Agriculture and Agri-Food Canada, 107 Science Place, \\ Saskatoon, SK, S7N 0X2, Canada \\ ${ }^{2}$ Department of Plant Sciences, University of Saskatchewan, 51 Campus Drive, Saskatoon, SK S7N 5A8, Canada \\ ${ }^{3}$ Western and Northern Service Centre, Parks Canada, 145 McDermot Avenue, Winnipeg, MB, R3B OR9, Canada
}

\begin{abstract}
Characterization of individual genetic variation within a plant population is an important component of plant conservation and evolutionary genetics. Here we present a marker-based approach to interpolating individual genetic information from a sample of plants to the whole population. The effectiveness of the approach was demonstrated by applying AFLP markers to estimate genetic distinctiveness from a sample of Festuca hallii (Vassey) Piper plants in six populations across the Canadian Prairies and proposing a linear interpolation to map the estimated genetic distinctiveness within each population. The interpolation was empirically verified for its effectiveness in one fescue population. Issues associated with and potential utility of the proposed interpolation are discussed.
\end{abstract}

Keywords: Genetic interpolation, genetic variation, genetic distinctiveness, plains rough fescue, AFLP, spatial genetics.

\section{INTRODUCTION}

Characterization of individual genetic variation within a plant population is an important component of plant conservation and evolutionary genetics [1]. It can yield a genetic census of each population member on identity, character and location and collectively, unique variation patterns may be emerged with respect to class, character and distribution. These genetic census data are useful not only for optimizing germplasm sampling strategies, but also for developing effective conservation strategies for a plant population at risk. Further exploration of these emerged patterns of individual variation in relation to population topography, soil, or environmental heterogeneity may yield insightful information for understanding and predicting population dynamics and evolution. However, little attention has been paid to such an individual characterization in plant populations [1].

Molecular markers have been successfully applied to assess genetic variation and structure among and within plant populations [2]. However, current genetic diversity analysis places emphasis on the overall level of genetic diversity and pattern of genetic structure and less on characterizations of individual variation $[1,3,4$,$] . Rapidly developing landscape$ genetics considering both landscape variables and genetic variation can provide help to characterize individual variation [5], but recent research efforts have focused largely on genetic connectivity among populations and genetic associations with landscape or environmental variables [6, 7]. Mapping geographic distributions of genetic variation has been made at the species level $[8,9]$, but not within a population

*Address correspondence to this author at the Plant Gene Resources of Canada, Saskatoon Research Centre, Agriculture and Agri-Food Canada, 107 Science Place, Saskatoon, SK, S7N 0X2, Canada; Tel: +1 306-9567642; Fax: +1 306-956-7246; E-mail: fuy @agr.gc.ca
[10]. Spatial analyses of individual genetic variation have been performed to address mainly genetic relatedness [11, 12] and distance [13], but not for a detailed characterization of individual variation [14]. Thus, a specific analysis of individual genetic variation appears to be warranted for conservation and evolutionary inferences of plant populations.

Recently, a new measure of genetic distinctiveness was introduced to identify genetically unique accessions from diverse crop germplasm collections [15]. This has been applied toward the expansion of the narrow genetic basis of improved crop gene pools $[15,16]$. Genetic distinctiveness is estimated with a modified Euclidian metric as the average dissimilarity of an accession against the other accessions of interest as reflected by their DNA fingerprinting profiles [15]. Such a molecular estimate, although limited to resolve genetic relatedness, may offer a useful means of characterizing individual plants in a population, as individual distinctiveness could be a good indicator of genetic diversity [17]. We reason that mapping individual genetic distinctiveness within a population would enhance conservation efforts in optimizing sampling strategies for germplasm conservation and utilization, identifying unique genotypes, defining local genetic hotspots, and monitoring diversity changes. These efforts may enhance germplasm conservation and habitat restoration by native grass seeding $[18,19]$ and facilitate the development of plant conservation strategies $[1,20]$.

Plains rough fescue [Festuca hallii (Vasey) Piper] is a native, tetraploid $(2 n=4 x=28)$, probably outcrossing, perennial species in the Fescue Prairie region of North America [21]. Due largely to agricultural conversion, Fescue Prairie has been declining over the last 100 years and currently occupies less than $15 \%$ of its original extent [22]. Much of what remains of fescue grassland occurs in small isolated patches, surrounded by cultivation or hay fields. Efforts have 
been made to protect Fescue Prairie remnants within national parks, provincial parks and provincial natural areas, but little is known about the genetic status of these protected populations. Recently, a genetic diversity analysis of fescue plants using the amplified restriction fragment polymorphism (AFLP) technique [23] was conducted with the hope to facilitate the management and conservation of these fragmented populations $[22,24]$.

Here we explore a marker-based approach to interpolating individual genetic information within a plant population. Specifically, we apply AFLP markers to estimate genetic distinctiveness from a sample of $F$. hallii plants in six populations across the Canadian Prairies and propose a linear interpolation to map the genetic distinctiveness of individual plants within each population. We also assess empirically the effectiveness of the linear interpolation in one fescue population and discuss the issues associated with and the potential utility of the proposed interpolation.

\section{MATERIALS AND METHODS}

\section{Interpolating Genetic Variation in a Natural Population}

For a given plant population with a defined population boundary, a representative sample of size $n$ individual plants is randomly selected within the boundary. The locations of the selected plants are recorded using a global positioning system (GPS). The seeds or leaf tissues of the selected plants are collected, depending on the biological features of plants and the type of molecular technique used for genotyping. Relative genetic distinctiveness of individual selected plants is estimated by average dissimilarity reflected in their DNA fingerprinting profiles as described below. Estimated genetic distinctiveness is interpolated over the defined population boundary using an interpolation method that takes into account both genetic information and spatial coordinates. Application of this approach requires specific consideration of the size of sampling, the choice and number of molecular markers, and the selection of an interpolation method.

The genetic distinctiveness of a plant can be estimated with a modified Euclidian metric as the average dissimilarity of the plant against the other plants of interest as reflected by their DNA fingerprinting profiles [15]. Using AFLP markers as an example, the similarities of a sampled plant with the other plants assayed can be calculated using the simple matching coefficient [25] as: $\mathrm{S}_{i j}=(a+d) /(a+b+c+d)$, where $\mathrm{S}_{i j}$ is the AFLP similarity between the plant $i(i=1$ to $n)$ and the other plant $j[j=1$ to $(n-1)], a$ is the number of AFLP bands shared in both $i$ and $j, b$ is the number of bands present in $i$ but not shared in $j, c$ is the number of bands present in $j$ but not shared in $i$, and $d$ is the number of bands absent from both $i$ and $j$. The AFLP dissimilarity for each pair of plants can be defined as $1-S_{i j}$. The average AFLP dissimilarity for the plant $i$ can be obtained by averaging all of the $n$ - 1 AFLP dissimilarities with which that plant was associated. This average dissimilarity measures the overall genetic difference present between the plant $(i)$ of interest and the remaining plants assayed. A higher average dissimilarity obtained from unlinked markers means that the plant has a genetic background more distinct from the other plants. This average dissimilarity can also be similarly estimated from random amplified polymorphic DNA (RAPD), microsatellite or other frequently applied molecular markers including DNA sequence variation.

There are numerous methods and software packages available for interpolation and smooth surface fitting of irregularly distributed data points [5, 26]. Commonly used methods in spatial analysis include linear, bivariate and spline interpolations [26]. The choice of interpolation methods for mapping genetic distinctiveness depends largely on the magnitude and nature of genetic autocorrelations across space. Given that few studies have interpolated scattered genetic data at an individual level [27], an assessment of the effectiveness of available interpolations is needed to select an interpolation method.

\section{Case Application to $F$. hallii Populations}

The genetic diversity analysis of $F$. hallii plants in six fescue populations in Manitoba and Saskatchewan was made in 2005 using AFLP technique. Three AFLP primer pairs were applied to screen approximately 60 samples randomly selected from each population. A total of 330 polymorphic AFLP bands were scored for each sample. Detailed site information, sampling method, and applied procedures for AFLP analysis and diversity analysis have been described in [22]. As the location of each selected sample was recorded with GPS coordinates, this data offers a case to demonstrate the effectiveness of interpolating genetic distinctiveness within these natural stands. The genetic distinctiveness of a sample compared to the other samples within a population was estimated as described above and compiled with summary statistics. This was done for each population using a SAS program written specifically for this application in SAS IML [28]. Test for normality of dissimilarity estimates in each population was made using the Shapiro-Wilk test of small samples, and significance of differences in the population means of average AFLP dissimilarity was tested using the unpaired $t$ test.

Four interpolation methods (i.e., linear, bivariate, spline and partial spline interpolations) available in the SAS package were evaluated with respect to accuracy and precision. These methods are described in detail in the SAS G3GRID procedure [28]. Cross-validation was performed for each method in each population by omitting 10 samples randomly selected from the original data set. Based on the remaining samples, the interpolation of the estimated genetic distinctiveness was made for a population according to the SAS G3GRID procedure with a given interpolation option. Differences between their derived and estimated values of the omitted 10 samples were recorded. As the G3GRID procedure does not provide a point prediction given the position of a sample, the derived distinctiveness of an omitted sample was obtained by generating the derived distinctiveness over a grid at the scale of $2000 \times 2000$ units corresponding to the whole area of a given population and searching the location closest to the omitted sample. The possible error distance with respect to the correct location of an omitted sample was smaller than $15 \mathrm{~cm}$ for these six populations, which is much smaller than the error of obtaining GPS coordinates for a selected plant in the field expected from a hand-held GPS instrument. This cross-validation was repeated 1000 times. The precision of point interpolations was assessed by three parameters as (1) the mean absolute difference between the 
interpolated and the true values, (2) the standard deviation of these differences, and (3) the root mean square error (RMSE) (described in [29]). This assessment was performed for each method and each population using a SAS program written specifically for this application in SAS IML.

The effects of variable sample size on the precision and accuracy of point interpolation were also evaluated using the same cross-validation procedure as above with modifications of the SAS program for the following factors. First, only the linear interpolation was applied, as it yielded more precise interpolations for these populations. Second, three sample sizes $(30,40$, approximately 50$)$ were used to interpolate, as 10 random samples were used for validation in each assessment. The same three parameters were estimated as for the assessment of interpolation methods. Note that a fixed number (i.e., 10) of samples were arbitrarily applied to makes the accuracy assessments compatible across the interpolation methods and sample sizes assessed.

The patterns of fine-scale genetic structure within a population were assessed using the software of GenAlEx v6 [30]. The Mantel test analysis was performed to evaluate any significant correlation of genetic distance with geographic distance in each population. The spatial genetic autocorrelation analysis was made to detect microspatial autocorrelations of fescue plants in each population. As the average physical distances of the pairwise plants sampled within six populations varied greatly from 29 to 114 meters with an average of 71 meters, the autocorrelation analysis was arbitrarily limited to a maximum distance of 90 meters with 30 equal distance classes.

The estimates of individual genetic distinctiveness were interpolated over the population boundary surveyed using the SAS G3GRID procedure with the linear interpolation option. As the population boundary was not defined originally, a rectangular shape was arbitrarily applied according to the recorded GPS coordinates for each population for ease of interpolation and illustration. The derived genetic distinctiveness was generated for each population at the scale of $1000 \times 1000$ units to an output file for the production of high resolution maps for use with other mapping programs (e.g., ArcGIS desktop software 9.2; [31]). For ease of comparison among populations, the derived distinctiveness was grouped into five distinctiveness classes of equal interval for each population. Counting the size of every distinctiveness class in a defined rectangle yielded an estimate of the total area of the distinctiveness map for each distinctiveness class.

\section{Empirical Verification of Mapping Distinctiveness}

An empirical assessment was made to verify the derived genetic distinctiveness. Because of its convenient location, the Kernen population nearby to the City of Saskatoon was selected. Based on the distinctiveness map and with the help of a hand-held GPS instrument (Trimble® GeoXTTM with real-time DGPS correction of sub-meter accuracy; Trimble Corp., Sunnyvale, CA), 24 individual plants located on the local areas with high or low derived distinctiveness (i.e., the top or low two classes of derived values, respectively) were randomly selected (12 individuals x 2 types of area). Note that these selected plants were largely a few meters apart from those plants sampled previously to establish the distinctiveness map (Fig. 3). The young tillers of the selected plants were collected in July 2007 and freeze-dried for DNA analysis. DNA extraction, AFLP analysis and data analysis were made following the same procedures of the previous diversity assessment that were described in [22]. However, the average dissimilarity of individual plants was estimated only among these 24 individual samples and compared between two sets of 12 samples to determine if those samples from the local areas with high derived distinctiveness are still more genetically unique than those from the local areas with low derived distinctiveness. The analysis of molecular variance (AMOVA; [32]) was also performed to assess the proportion of the total AFLP variation resided between the two types of samples and its significance by 10000 random permutations.

\section{RESULTS}

The estimates of individual average AFLP dissimilarity within each population were summarized in Table 1. The Shapiro-Wilk tests showed that these estimates were normally distributed, except in the Turtleford population with a marginal non-normality $(P<0.04)$ with a skewness of 0.47 toward larger estimates. The population means of average AFLP dissimilarity ranged from 0.2565 for Riding Mountain to 0.2843 for Turtleford (Table 1), but their differences were not statistically significant, based on the unpaired $t$ tests.

Evaluation of the four interpolation methods showed that the linear interpolation appeared to be the best in mapping genetic distinctiveness with respect to accuracy and precision, followed by spline, bivariate and partial spline. The linear interpolation consistently generated the smallest esti-

Table 1. Summary statistics of genetic distinctiveness estimated by the average AFLP dissimilarity of an individual plant against the other plants collected in one of six Festuca hallii populations in Manitoba (MB) and Saskatchewan (SK)

\begin{tabular}{|c|c|c|c|c|c|}
\hline Population & Sample size & Mean & Standard & Minimum & error \\
\hline Batoche, SK & 59 & 0.2668 & 0.0380 & 0.2516 & 0.2896 \\
\hline Kernen, SK & 60 & 0.2690 & 0.0442 & 0.2495 & 0.2483 \\
\hline Macrorie, SK & 59 & 0.2742 & 0.0540 & 0.2476 & 0.2937 \\
\hline Prince Albert, SK & 58 & 0.2716 & 0.0559 & 0.2584 & 0.3035 \\
\hline Riding Mountain, MB & 58 & 0.2565 & 0.0547 & 0.386 \\
\hline Turtleford, SK & 60 & 0.2843 & 0.3129 \\
\hline
\end{tabular}


mated mean, standard deviation and RMSE in all of the populations surveyed (Table 2). Evaluation on the influences of the three sample sizes on distinctiveness interpolation revealed that larger sample sizes marginally increased the performance of the linear interpolation, as reflected by smaller estimated mean, standard deviation and RMSE in all of the populations surveyed (not shown), except in the Macrorie population. Given our sample size of approximately 60 , the mean difference between the derived and estimated distinctiveness ranged from 0.008 for Batoche to 0.014 for Turtleford, reflecting about $5 \%$ or less of the estimated average dissimilarities (Table 2).

Significant genetic autocorrelations at a short distance were observed in the four populations (approximately 3 me- ters in Batoche, Kernen and Riding Mountain populations; approximately 6 meters in the Prince Albert population). However, the number of individual pairs within these significant distance classes was small, ranging from 7 to 11 over the four populations. For illustration, Fig. (1) shows the genetic autocorrelation coefficient $r$ observed as a function of distance $(\mathrm{m})$ in the Kernen and Prince Albert populations. Mantel tests revealed a significant, but weak, correlation $(r=$ $0.13, P<0.001)$ of genetic distance with geographic distance only in the Batoche population.

The six fescue populations showed different landscapes of genetic distinctiveness (Figs. 2 and 3). When the derived distinctiveness was categorized into five classes, each class differs in size (or area) within and among the maps (Table

Table 2. Precision comparison of point interpolations of estimated genetic distinctiveness over six Festuca hallii populations based on linear, bivariate, spline and partial spline interpolation methods. RMSE is the root mean square error

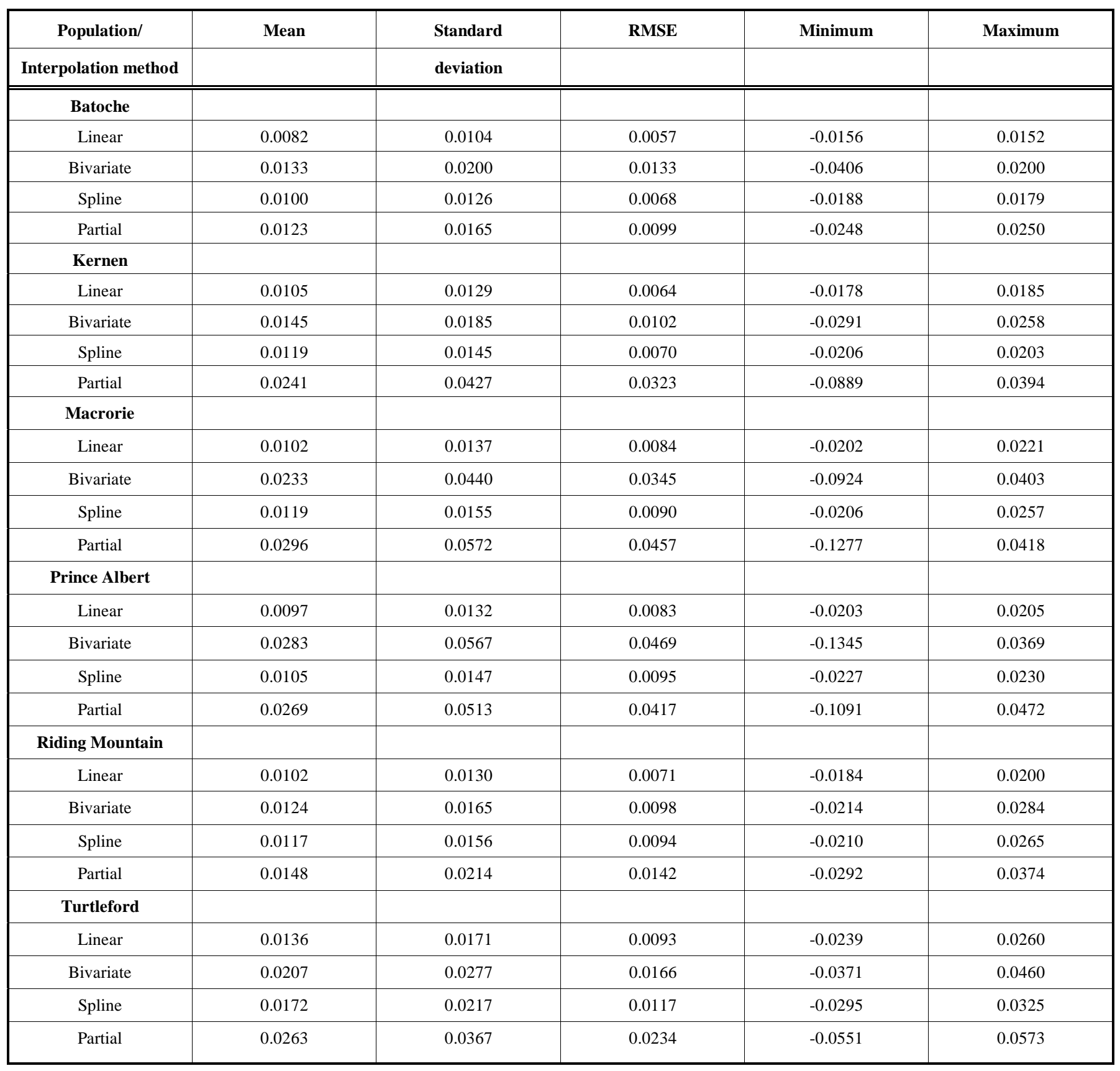



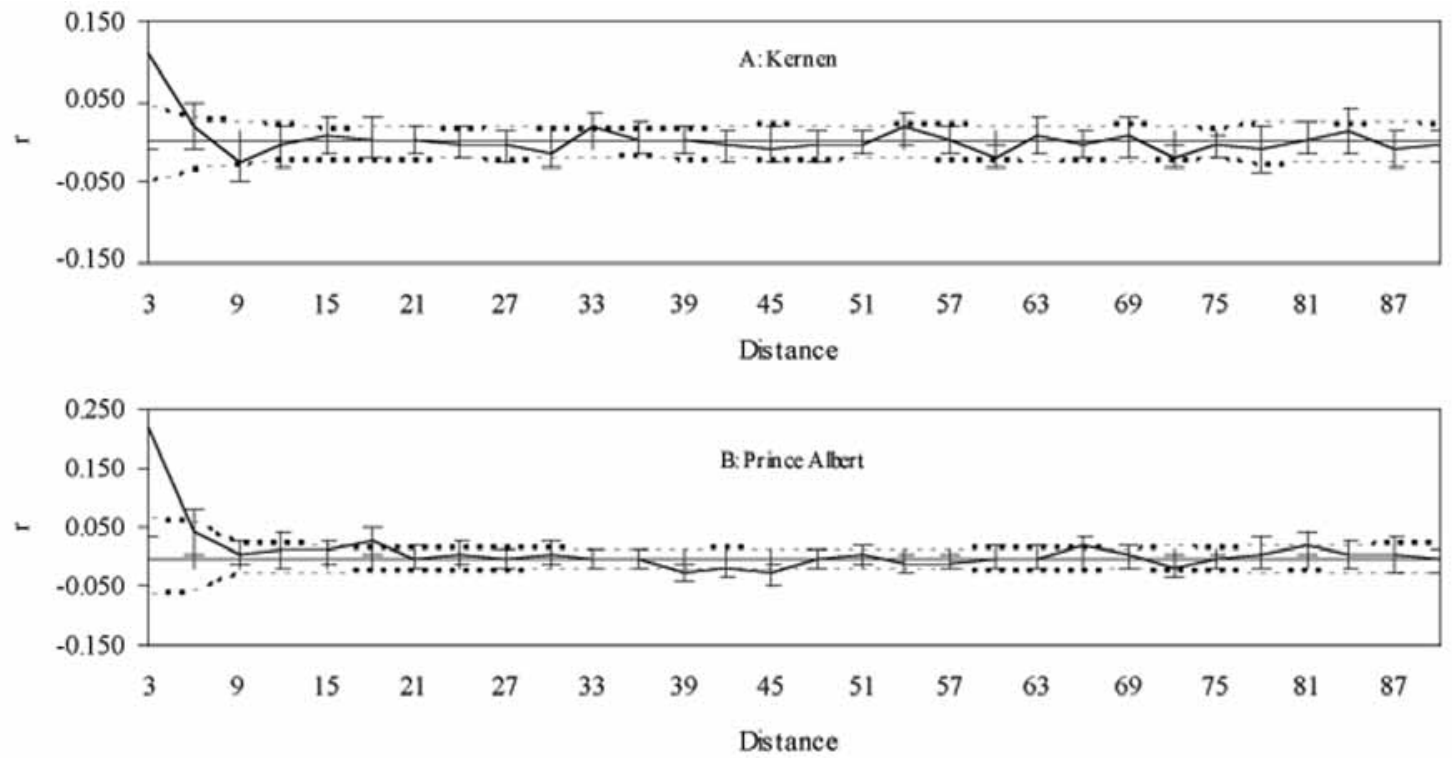

Fig. (1). Correlograms showing the genetic autocorrelation coefficient $r$ as a function of distance (m), 95\% CI about the null hypothesis of a random distribution of Festuca hallii plants, and 95\% confidence error bars about $r$ as determined by bootstrapping, in the Kernen and Prince Albert populations.

3). For example, the Macrorie population has a relatively large area $(9.22 \%)$ of fescue plants found with the highest derived distinctiveness values, followed by the Turtleford (4.43\%), Riding Mountain (2.77\%), Prince Albert (0.84\%), Kernen $(0.76 \%)$, and Batoche $(0.54 \%)$ populations. The local areas for each class also differ in shape and were widely spread. For example, the highly unique genotypes resided over various map areas as displayed in the Batoche and Turtleford populations (Fig. 2). However, many large areas with one distinctiveness class were also observed in each population, mainly due to the inadequate sampling coverage. For example, one large area on the eastern edge of the Kernen map was interpolated with the second top class of derived distinctiveness (Fig. 3).

With the maps in UTM projection, one can easily locate the position of a plant with the derived distinctiveness in a population using a hand-held GPS instrument (Fig. 3). Empirical assessment in the Kernen population yielded encouraging results as summarized in Table 4. The samples from the local areas with high derived distinctiveness were more genetically unique than those from the local areas with low derived distinctiveness, as reflected in two different means of average dissimilarity (Table 4). The samples from the local areas with high derived distinctiveness captured about $2 \%$ more AFLP variation than those from the local areas with low derived distinctiveness, as obtained from the analysis of molecular variance (Table 4). This difference was reflected in both the number of polymorphic AFLP bands and the mean band frequency (Table 4). For example, the samples expected for high distinctiveness had 128 polymorphic bands with a mean band frequency of 0.38 , while those samples expected for low distinctiveness had 124 polymorphic bands with a mean band frequency of 0.35 .

\section{DISCUSSION}

We have explored a marker-based approach to interpolating individual genetic information from a sample of plants to the whole population and empirically demonstrated the ef- fectiveness of this approach for mapping genetic distinctiveness within six plains rough fescue populations across the Canadian Prairies. Although the demonstration is technically far from ideal as discussed below, the effectiveness of interpolation found in the fescue populations (Tables $\mathbf{2}$ and $\mathbf{4}$ ) appears to be encouraging for its applications to other plant populations. The availability of abundant molecular markers and sophisticated spatial analysis tools may make the interpolation application more feasible and effective. Thus, the proposed interpolation could provide an alternative means for characterizing individual genetic variation, particularly within protected populations of a plant species with a mixedmating system or clonal propagation.

\section{Issues and Limitations Associated with Mapping Genetic Variation}

The proposed interpolation is equally applicable to many other measures of individual genetic variation such as individual relatedness, but why genetic distinctiveness was applied in the study? The distinctiveness measure, unlike many relatedness measures currently available [33], is not limited to the type of molecular markers used and the ploidy level of a plant species assessed. Also, the genetic distinctiveness, although related to genetic relatedness measure, carries more information on genetic diversity, less on genetic association as by genetic relatedness. Thus, a distinctiveness map is at least practically feasible and probably more informative for diversity inferences such as optimizing sampling strategies for germplasm conservation and utilization, identifying unique genotypes, defining local genetic hotspots, or monitoring diversity changes. However, this should not downplay the usefulness of interpolating individual relatedness or other diversity estimates for other workable plant species, as derived relatedness or diversity maps may be more informative for population dynamic analyses such as gene flow and genetic drift. 

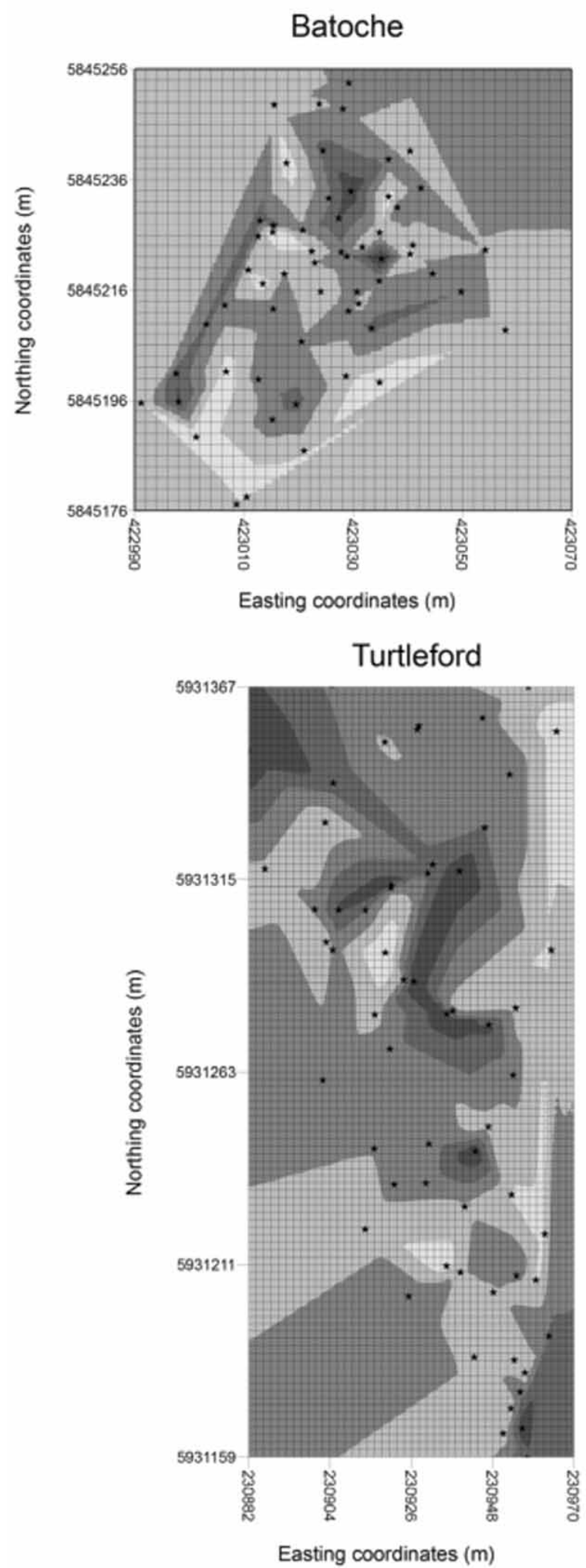
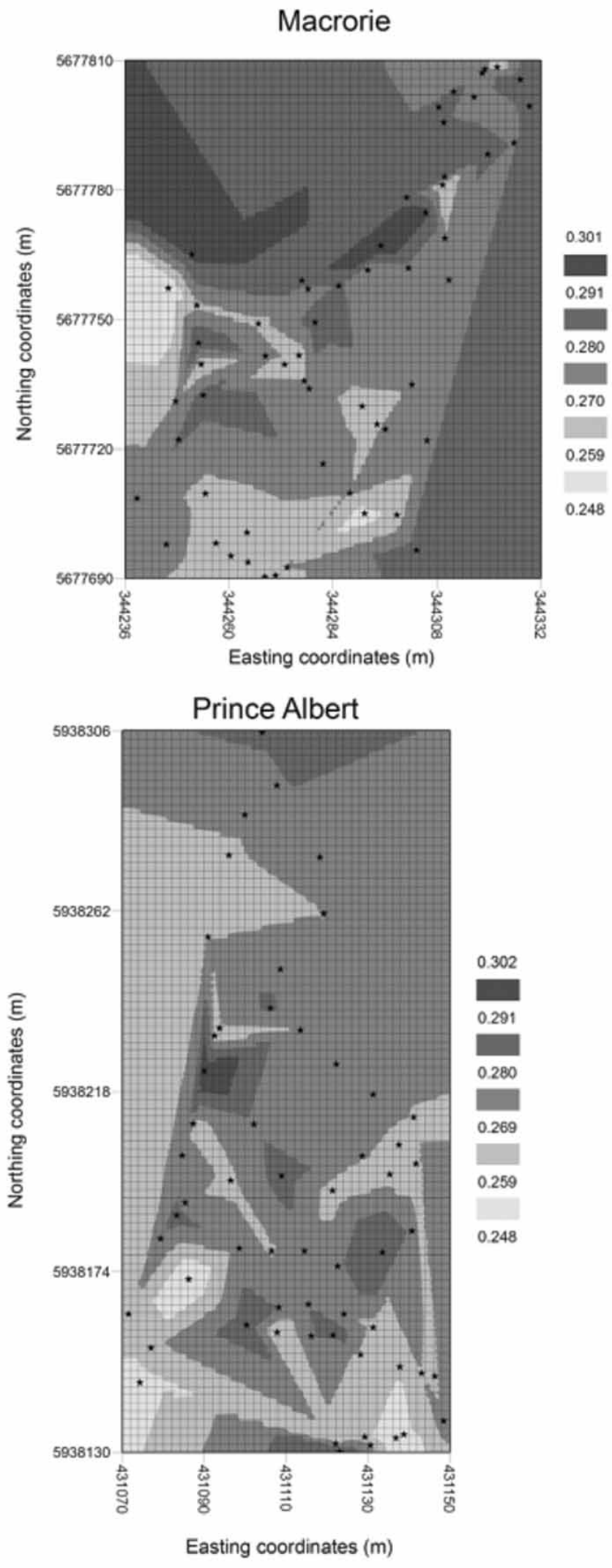

Fig. (2). Illustrative interpolations of estimated genetic distinctiveness of Festuca hallii plants in four populations (Batoche, Macrorie, Turtleford, and Prince Albert) surveyed in Saskatchewan. Note that scales in both coordinates (in UTM) and estimated distinctiveness differ for four populations. Derived values of distinctiveness are grouped into five distinctiveness classes for ease of comparison among populations. Darker areas of the map represent the possible locations of plants with higher derived distinctiveness. The positions of the original plants sampled for interpolation are shown with filled stars. 

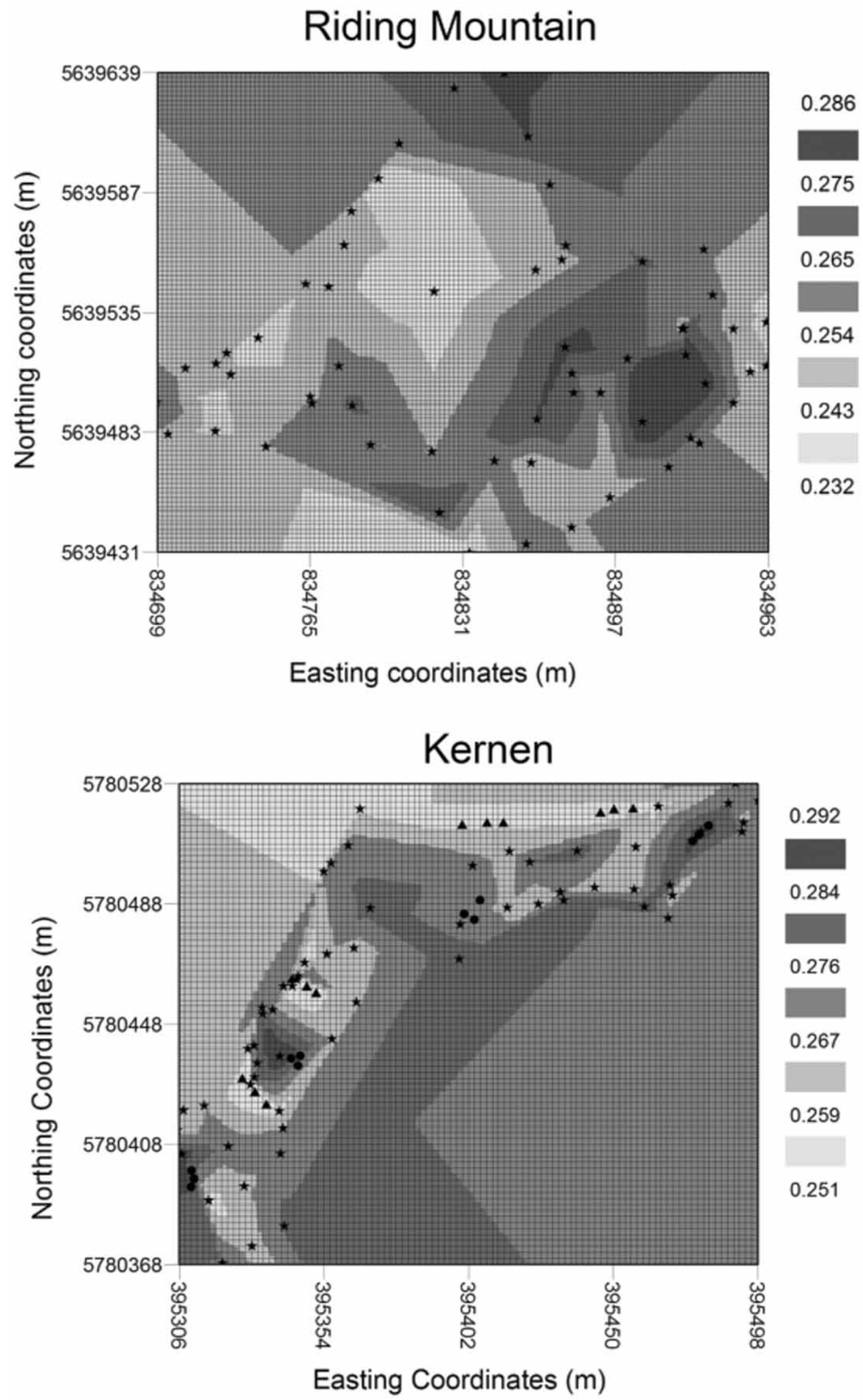

Fig. (3). Expanded illustration of point interpolations of estimated genetic distinctiveness of Festuca hallii plants in the Riding Mountain and Kernen populations. Note that scales in both coordinates (in UTM) and estimated distinctiveness differ for two populations. Derived values of distinctiveness are grouped into five distinctiveness classes. Darker areas of the map represent the possible locations of plants with higher derived distinctiveness. The positions of the original plants sampled for interpolation are shown with filled stars. The Kernen map also shows the positions of the plants sampled for the empirical interpolation verification. Twelve plants selected randomly from the local areas with low or high derived distinctiveness are labelled with filled triangles and circles, respectively. 
Table 3. The proportions of the map area for five distinctiveness classes estimated for six Festuca hallii populations

\begin{tabular}{|c|c|c|c|c|c|}
\hline \multirow[t]{2}{*}{ Population } & \multicolumn{5}{|c|}{$\begin{array}{l}\text { Proportion of the map area for each class of derived } \\
\text { distinctiveness values from high to low }{ }^{\text {a }}\end{array}$} \\
\hline & $\mathbf{I}$ & II & III & VI & $\mathbf{V}$ \\
\hline Batoche & 0.54 & 4.51 & 32.27 & 57.23 & 5.44 \\
\hline Kernen & 0.76 & 24.51 & 46.19 & 20.67 & 7.86 \\
\hline Riding Mountain & 2.77 & 15.52 & 40.43 & 31.59 & 9.67 \\
\hline Turtleford & 4.43 & 11.37 & 46.66 & 33.67 & 3.86 \\
\hline
\end{tabular}

${ }^{a}$ The five classes have an equal interval of derived distinctiveness in each population, but the intervals and the total ranges of derived values differ among six populations. See Figs. ( 2 and 3 ) for the scale of each class in each population.

There are several obvious limitations in both the measurement of genetic distinctiveness and the choice of an interpolation method. Average dissimilarity so estimated can recognize the genetic distinctiveness, but not necessarily the genetic relatedness, of individual plants [15]. For example, two closely related plants that are genetically distinct from the remaining population could have similar higher levels of dissimilarity than the other plants and both plants would have been identified as genetically distinct plants. If the molecular markers used are selectively neutral, the genetic distinctiveness so obtained may not reflect directly the adaptability (or fitness) of a plant. This could reflect another weakness of mapping distinctiveness for germplasm utilization. As genetic autocorrelations of individual plants are likely unknown prior to an experiment, the effectiveness of an interpolation method to be applied is not certain, requiring additional efforts of assessment. Specific consideration is also required for the sampling effort, the choice and number of molecular markers, and the interpolation method, thus adding more uncertainties to the effectiveness of mapping genetic distinctiveness. Some of these limitations are also expected for individual relatedness interpolation.

The proposed interpolation would perform better in a plant species with a mixed-mating system and/or clonal propagation than the outcrossing fescue plants with high gene flow, as the former generally harbors a strong genetic autocorrelation. Thus, it is not surprising to find only small genetic patches (or neighboring sizes within a few meters apart) in fescue plants, which may reflect the effect of plant propagations with seeds and short rhizomes. The small patches observed are consistent with the reports of weak local spatial structure in several plant species [34], and explain well the effectiveness of the linear interpolation method used in mapping the genetic distinctiveness. Similarly, a patch size of one meter or less was observed for flower and stem morphs in Impatiens pallida and I. capensis populations [35]. However, a large patch size of Douglas-fir trees up to 20 meters apart was inferred from tree heights at ages from 6 to 12 years [36]. Thus, a proper assessment of interpolation errors is needed, because an interpolation method may not take into account all the existing spatial structures of errors as illustrated in laser scanning data [29, 37]. Also, an interpolation error map may be desirable for some characterizations.

\section{Improvement of Mapping Genetic Variation}

This study represents our first attempt to map individual genetic variation within a population. Further application and modification, particularly with individual genetic relatedness, are still needed to enhance its effectiveness for plant populations of other species. The average dissimilarity as we obtained in this study may differ among and within populations, depending on many factors such as the number of molecular markers and samples assayed. Increasing unlinked markers to cover the plant genome and sampling more individual plants from a population would enhance the estimation of genetic distinctiveness. For example, the population mean of average dissimilarities in the Kernen population was 0.269 with 60 samples, but ranged from 0.328 to 0.352 with 12 samples in the verification assay (Tables 1 and 4). Thus, the estimates of genetic distinctiveness are limited and relative only to those assayed in a particular study. Caution is warranted in data interpretation [15]. The choice of a molecular marker technique to be applied may also depend on a few factors such as plant biology, operational feasibility and experimental costs, but the ability to generate many unlinked markers to adequately sample the plant genome is among the important factors to be considered. The effects of variable sample sizes on fescue distinctiveness interpolations as found in this study appear to be relatively small, but adequate sampling coverage of a study site is an important issue. Many large areas with the same levels of derived distinctiveness were observed in each population, which reflects the fact that the original sampling of these sites did not cover well the population boundary (defined after the fact as a rectangle). The derived distinctiveness in these areas had large interpolation errors due to inadequate sampling. Systematic sampling if practically feasible may be more appropriate than random sampling [26].

There are many free or commercial computer programs currently available that can perform interpolation and map production [5]. We applied the SAS G3GRID procedure to interpolate genetic variation and the ArcGIS ArcMap routine to produce two-dimension maps, largely due to the extensive usages of these programs and for ease of illustration. In fact, both programs can perform both functions, although operational complexity and map quality differ. In our view on the management of a population, a two-dimension map may be more informative than a three-dimension map. There also are 
Table 4. Comparison of AFLP variability between two sets of Festuca hallii samples representing the local areas mapped with the most or least unique genotypes in the Kernen population

\begin{tabular}{|c|c|c|}
\hline Mapped area & Most unique $^{\text {a }}$ & Least unique $^{12}$ \\
\hline \hline Sample size & 128 & 124 \\
\hline Number of polymorphic bands scored & 0.38 & 0.35 \\
\hline Mean frequency of scored bands & $95.32^{\text {ns }}$ & 42.00 \\
\hline Heterogeneity $\chi^{2}$-test value for all bands & 48.85 & $1.96 *$ \\
\hline Variation within each set of samples & $0.3524 *$ & 0.3280 \\
\hline Variation between two sets of samples (\%) & $0.3018-0.4004$ & $0.2996-0.3508$ \\
\hline Mean of average dissimilarities (ADs) & & \\
\hline Range of ADs & & \\
\hline
\end{tabular}

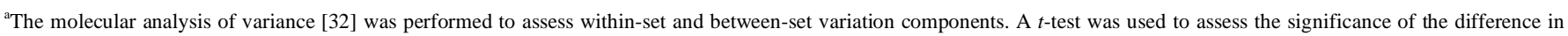
average dissimilarity between two sets of samples. Significance of a test is labeled with $*$ at $P<0.05$ and ${ }^{\text {ns }}$ at $P>0.05$.

many computer programs specialized in spatial analysis of genetic variation, including SPAGEDI [38], Geneland [39] and Alleles in Space [27]. These programs were not designed to map genetic distinctiveness and their mapping products may be lower in quality (with different colours) for management of a plant population at risk. Thus, specific integrated software to process distinctiveness or relatedness estimation, interpolation and map production is desired for interpolating plant genetic variation.

\section{Implications for Plains Rough Fescue Conservation and Utilization}

How to utilize these newly created maps of genetic variation effectively with respect to the management and conservation of plant populations is beyond the intention of this article and remains an interesting issue to be explored. However, it is obvious that the distinctiveness maps obtained in this study appear to be useful in several aspects of plains rough fescue conservation and utilization for habitat restoration. First, the distinctiveness maps could enhance germplasm sampling for habitat restoration by capturing more genetically diverse genotypes [18]. For example, sampling maternal genotypes (or plant tillers) from the local areas with high derived distinctiveness in the Kernen population would yield $2 \%$ more AFLP variation than the sampling done from the local areas with low derived distinctiveness (Table 4). However, producing distinctiveness maps solely for genetic sampling in many unprotected populations may not necessarily be cost-effective. Second, the distinctiveness maps could be used to locate unique genotypes in the three populations (Prince Albert, Batoche, Riding Mountain) currently under protection at national parks, identify the local areas with high derived distinctiveness for multiple genetic sampling for habitat restoration, configure the proportion of the local areas with low derived distinctiveness needed for re-seeding with desirable genotypes, or monitor the diversity changes over time in the protected populations. For example, there are larger, but fewer, local areas with the lowest derived distinctiveness in the Riding Mountain park, while the Batoche population displayed smaller, but more, local areas with the lowest derived distinctiveness (Fig. 2), suggesting variable effort is required for re-seeding with desirable genotypes in these protected stands. Third, our direct observation appears to suggest that the obtained landscapes of genetic distinctiveness did not match with the site topography (Figs 2 and 3), but could be associated with the soil and environmental heterogeneity of each site. Further critical empirical assessments on these associations not only may shed some light on the factors responsible for shrinking habitat patch sizes in these fescue remnants, but also could yield insightful information for understanding evolutionary processes.

\section{ACKNOWLEDGEMENTS}

We are grateful to David Giffen for his assistance in map production and John Avise, Daniel Schoen, Walter Willms, and anonymous reviewers for their constructive comments on the early version of the manuscript. Part of the research was financially supported by Parks Canada Ecological Integrity Innovation and Leadership Fund.

\section{REFERENCES}

[1] Avise, J.C. The history, purview, and future of conservation genetics. In Conservation Biology: Evolution in Action; Carroll, S.P.; Fox, C.W. Eds.; Oxford University Press, Oxford, UK, 2008; (in press).

[2] Haig, S.M. Molecular contributions to conservation. Ecology, 1998, 79: 413-425.

[3] Karp, A. The new genetic era: will it help us in managing genetic diversity? In Managing Plant Genetic diversity; Engels, J.M.M.; Rao, V.R.; Brown, A.H.D.; Jackson, M.T.; Eds.; International Plant Genetic Resources Institute, Rome, Italy, 2002; pp. 43-56.

[4] Nybom, H. Comparison of different nuclear DNA markers for estimating intraspecific genetic diversity in plants. Mol. Ecol., 2004, 13: 1143-1155.

[5] Storfer, A.; Murphy, M.A.; Evans, J.S.; Goldberg, C.S.; Robinson, S.; Spear, S.F.; Dezzani, R.; Delmelle, E.; Vierling, L.; Waits, L.P. Putting the 'landscape' in landscape genetics. Heredity, 2007, 98: 128-142.

[6] Miller, M.P.; Bellinger, M.R.; Forsman, E.D.; Haig, S.M. Effects of historical climate change, habitat connectivity, and vicariance on genetic structure and diversity across the range of the red tree vole (Phenacomys longicaudus) in the Pacific Northwestern United States. Mol. Ecol., 2006, 15: 145-159.

[7] Manel, S.; Berthoud, F.; Bellemain, E.; Gaudeul, M.; Luikart, G.; Swenson, J.E.; Eaits, L.P.; Taberlet, P.; Intrabiodiv Consortium. A new individual-based spatial approach for identifying genetic discontinuities in natural populations. Mol. Ecol., 2007, 16: 20312043. 
[8] Ferguson, M.E.; Ford-Lloyd, B.V.; Robertson, L.D.; Maxted, N.; Newbury, H.J. Mapping the geographical distribution of genetic variation in the genus Lens for the enhanced conservation of plant genetic diversity. Mol. Ecol., 1998, 7: 1743-1755.

[9] Guarino, L.; Jarvis, A.; Hijmans, R.J.; Maxted, N. Geographic information systems (GIS) and the conservation and use of plant genetic resources. In Managing Plant Genetic diversity; Engels, J.M.M.; Rao, V.R.; Brown, A.H.D.; Jackson, M.T.; Eds.; International Plant Genetic Resources Institute, Rome, Italy, 2002; pp. 387-403.

[10] Greene, S.L.; Gritsenko, M.; Vandemark, G.; Johnson, R.C. Predicting germplasm differentiation using GIS-derived information. In Managing Plant Genetic diversity; Engels, J.M.M.; Rao, V.R.; Brown, A.H.D.; Jackson, M.T.; Eds.; International Plant Genetic Resources Institute, Rome, Italy, 2002; pp. 404-412.

[11] Loiselle, B.A.; Sork, V.L.; Nason, J.; Graham, C. Spatial genetic structure of a tropical understory shrub, Psychotria officinalis (Rubiacease). Amer. J. Bot., 1995, 82: 1420-1425.

[12] Ritland, K. Estimators for pairwise relatedness and individual inbreeding coefficients. Genet. Res., Cambridge, 1996, 67: 175-185.

[13] Rousset, F. Genetic differentiation between individuals. J. Evol. Biol., 2000, 13: 58-62.

[14] Elmer, K.R.; Dávila, J.A.; Lougheed, S.C. Applying new interindividual approaches to assess fine-scale population genetic diversity in a neotropical frog, Eleutherodactylus ockendeni. Heredity, 2007, 99: 506-515.

[15] $\mathrm{Fu}$, Y.B. Genetic redundancy and distinctness of flax germplasm as revealed by RAPD dissimilarity. Plant Genet. Resour., 2006, 4: 117-124.

[16] Fu, Y.B.; Peterson, G.W.; Morrison, M.J. Genetic diversity of Canadian soybean cultivars and exotic germplasm revealed by simple sequence repeat markers. Crop Sci., 2007, 47: 1947-1954.

[17] Hedrick, P.W. Genetics of Populations (Third Edition), Jones and Bartlett: Boston, MA, 2005.

[18] Fu, Y.B.; Phan, A.T.; Coulman, B.; Richards, K.W. Genetic diversity in natural populations and corresponding seed collections of little bluestem as revealed by AFLP markers. Crop Sci., 2004, 44: 2254-2260.

[19] Richards, C.M.; Antolin, M.F.; Reilley, A.; Poole, J.; Walters, C. Capturing genetic diversity of wild populations for ex situ conservation: Texas wild rice (Zizania texana) as a model. Genet. Resour. Crop Evol., 2007, 54: 837-848.

[20] Schaal, B.; Leverich, W.J. Conservation Genetics: theory and practice. Ann. Missouri Bot. Garden, 2005, 92: 1-11.

[21] Anderson, D.G. Festuca hallii (Vasey) Piper (Hall's fescue): a technical conservation assessment. [online]. USDA Forest Service, Rochy Mountain Region, Fort Collins, CO, 2000, Available from www.fs.fed.us/r2/projects/scp/assessments/festucahallii.pdf [assessed 13 March 2008].

[22] Qiu, J.; Fu, Y.B.; Bai, Y.; Wilmshurst, J.F. Patterns of amplified restriction fragment polymorphism in natural populations and cor- responding seed collections of plains rough fescue. Can. J. Bot., 2007, 85: 484-492.

[23] Vos, P.; Hogers, R.; Bleeker, M.; Reijans, M.; van De Lee, T.; Hornes, M.; Frijters, A.; Peleman, J.; Kuiper, M.; Zabeau, M. AFLP: A new technique for DNA fingerprinting. Nucleic Acids Res., 1995, 23: 4407-4414

[24] Fu, Y.B.; Qiu, J.; Peterson, G.W.; Willms, W.D.; Wilmshurst, J.F. Characterization of microsatellite markers for rough fescue species (Festuca spp.). Mol. Ecol. Notes, 2006, 6: 894-896.

[25] Sokal, R.R.; Michener, C.D. A statistical method for evaluating systematic relationships. Univ. Kansas Sci. Bull., 1958, 38: 14091438.

[26] Fortin, M.-J.; Dale, M. Spatial analysis: a guide for ecologists, Cambridge University Press: Cambridge, UK, 2005.

[27] Miller, M.P. Allele in space: computer software for the joint analysis of interindividual spatial and genetic information. J. Hered., 2005, 96: 722-724.

[28] SAS Institute Inc. The SAS system for windows V8.02, SAS Institute Incorporated: Cary, NC, USA, 2004.

[29] Desmet, P.J.J. Effects of interpolation errors on the analysis of DEMs. Earth Surf. Proc. Landforms, 1997, 22: 563-580.

[30] Peakall, R.; Smouse, P.E. GENALEX 6: genetic analysis in Excel. Population genetic software for teaching and research. Mol. Ecol. Notes, 2006, 6: 288-295.

[31] ESRI. ArcGIS desktop software 9.2, ESRI: Relands, CA, USA, 2006.

[32] Excoffier, L.; Laval, G.; Schneider, S. Arlequin ver. 3.0: An integrated software package for population genetics data analysis. Evol. Bioinform. Online, 2005, 1: 47-50.

[33] Ritland, K. Multilocus estimation of pairwise relatedness with dominant markers. Mol. Ecol., 2005, 14: 3157-3165.

[34] Smouse, P.E.; Peakall, R. Spatial autocorrelation analysis of individual multiallele and multilocus genetic structure. Heredity, 1999, 82: 561-573.

[35] Schoen, D.J.; Latta, R.G. Spatial autocorrelation of genotypes in populations of Impatiens pallida and Impatiens capensis. Heredity, 1989, 63: 181-189.

[36] Fu, Y.B.; Yanchuk, A.D.; Namkoong, G. Spatial patterns of tree height variations in a series of Douglas-fir progeny trials: implications for genetic testing. Can. J. For. Res., 1999, 29: 714-723.

[37] Smith, S.L.; Holland, D.; Longley, P. Interpreting interpolation: the pattern of interpolation errors in digital surface models derived from laser scanning data. Centre for Advanced Spatial Analysis Working Paper Series 66, 2003.

[38] Hardy, O.J.; Vekemans, X. SPAGEDI: a versatile computer program to analyze spatial genetic structure at the individual or population levels. Mol. Ecol. Notes, 2002, 2: 618-620.

[39] Guillot, G.; Mortier, F.; Estoup, A. Geneland: a computer package for landscape genetics. Mol. Ecol. Notes, 2005, 5: 712-715. 